\title{
The Role of Dopamine in Intracranial Self-Stimulation of the Ventral Tegmental Area
}

\author{
H. C. Fibiger, F. G. LePiane, A. Jakubovic, and A. G. Phillips \\ Division of Neurological Sciences, Department of Psychiatry and Department of Psychology, The University of British \\ Columbia, Vancouver, B.C. V6T 2A1, Canada
}

The role of dopaminergic (DA) neurons in brain stimulation reward produced by electrical stimulation of the ventral tegmental area (VTA) was investigated in the rat. In the first experiment, extensive 6-hydroxydopamine lesions of the ascending fibers of the mesotelencephalic DA projections resulted in significant changes in intracranial self-stimulation (ICS) rate-current intensity functions when the lesion was ipsilateral to the stimulating electrode. Similar contralateral lesions had no effect on these functions, thus ruling out lesion-induced performance deficits as being responsible for the decreases in ICS rates across the wide range of current intensities that occurred after the ipsilateral lesions. In the second experiment, ICS obtained from electrodes in the VTA resulted in significant increases in the DA metabolites, 3,4-dihydroxyphenylacetic acid (DOPAC) and homovanillic acid (HVA) in the striatum, nucleus accumbens, and olfactory tubercle ipsilateral to the stimulating electrode. The ratios of DOPAC and HVA to DA, considered to be indices of DA utilization, were also increased in these brain regions ipsilateral to the electrode. No changes were observed in the contralateral striatum, nucleus accumbens, and olfactory tubercle. Similar increases were observed in stimulated "yoked" animals that received brain stimulation at identical rates and currents but did not lever-press for this stimulation. The third experiment examined the effects of lever-pressing for food on an FR8 schedule of reinforcement on DA utilization in the striatum, nucleus accumbens, and olfactory tubercle. Despite high rates of responding, no effects were observed on DOPAC:DA or HVA:DA ratios in these brain regions. In contrast, "yoked" animals that were subject to identical patterns of food delivery without lever-pressing showed significant increases in HVA:DA ratios in the nucleus accumbens.

The results of these experiments indicate that ICS obtained from electrodes in the VTA activates mesotelencephalic DA neurons and that these neurons mediate some of the rewarding properties of electrical stimulation obtained from this brain region. There was no indication that these neurons are activated during lever-pressing for food.

\footnotetext{
Received Nov. 17, 1986; revised June 15, 1987; accepted June 15, 1987

We thank Davina Fu and Stella Atmadja for their excellent technical assistance. Thanks also to Carolyn Szostak for her valuable assistance on the statistical analyses. This work was supported by the Medical Research Council of Canada (Program Grant 23).

Correspondence should be addressed to H. C. Fibiger, Division of Neurological Sciences, Department of Psychiatry, Unıversity of British Columbia, Vancouver, B.C. V6T $2 \mathrm{~A} 1$, Canada.

Copyright C 1987 Society for Neuroscience $0270-6474 / 87 / 123888-09 \$ 02.00 / 0$
}

Intracranial self-stimulation (ICS) can be obtained from numerous sites in the central nervous system (Phillips, 1984). There is considerable evidence that dopaminergic (DA) neurons are among the neural substrates that mediate the reinforcing properties of ICS obtained from at least some of these sites. For example, the robust ICS obtained from electrodes in the ventral tegmental area (VTA), a region rich in DA perikarya, is greatly reduced after selective lesions of the ascending projections of these neurons (Phillips and Fibiger, 1978). Similarly, ICS obtained from electrodes in the lateral hypothalamus is much reduced by lesions of the VTA (Koob et al., 1978). In the above instances, control procedures indicated that these reductions in ICS could not be attributed to the motor deficits that often accompany lesions of DA neurons. These and data from many other sources suggest that the mesolimbic D $\wedge$ projection is an important reward-related system (see Fibiger and Phillips, 1986, for review).

Despite the significant body of data pointing to an important role for DA neurons in brain-stimulation reward, some recent findings appear to be inconsistent with this hypothesis. For example, the electrophysiological characteristics of neurons that are stimulated by ICS electrodes in the medial forebrain bundle are different from what one would predict for DA neurons. Specifically, the critical neuronal substrate for ICS in the lateral hypothalamus and VTA appears to be a descending, myelinated pathway (Gallistel et al., 1981; Bielajew and Shizgal, 1982). In addition, Gallistel et al. (1985) have reported that metabolic activation of DA projection systems is not a necessary concomitant of ICS obtained from electrodes in the posterior hypothalamus or VTA. Given these results, the present experiments were undertaken to obtain further information on the role of DA neurons in ICS obtained from electrodes in the VTA.

\section{Experiment 1}

This experiment was designed to confirm and extend an earlier report of disruption of ICS from VTA electrodes following 6-hydroxydopamine (6-OHDA) lesions of the mesotelencephalic DA projections (Phillips and Fibiger, 1978). Instead of using only one stimulation current for each animal, a more sensitive measure of brain stimulation reward was obtained by generating response rate-stimulus intensity functions before and after the 6-OHDA lesions. A further refinement over the earlier study involved the use of unilateral lesions, either ipsilateral or contralateral to the stimulating electrode, as a control for performance deficits.

\section{Materials and Methods}

Male hooded rats of the Long-Evans strain (300-350 gm) were housed in individual stainless steel cages throughout the experiment. Food and 
Table 1. Concentrations of DA in striatum, nucleus accumbens, and olfactory tubercle following unilateral 6-OHDA lesions of the mesotelencephalic DA pathways (ng/mg protein, mean \pm SEM)

\begin{tabular}{|c|c|c|c|}
\hline & \multicolumn{3}{|l|}{ Dopamine } \\
\hline & \multicolumn{2}{|l|}{ Hemisphere } & \multirow{2}{*}{$\begin{array}{l}\text { Lesion } \\
\text { ( } \% \text { of } \\
\text { control) }\end{array}$} \\
\hline & Control & Lesion & \\
\hline \multicolumn{4}{|l|}{ Striatum } \\
\hline $\begin{array}{l}\text { Ipsilateral, mean } \pm \text { SEM } \\
\text { Contralateral, mean }+\end{array}$ & $123.8 \pm 2.9$ & $0.4 \pm 0.1$ & $0.3 \pm 0.0$ \\
\hline SEM & $100.9 \pm 7.0$ & $0.1 \pm 0.0$ & $0.1 \pm 0.0$ \\
\hline \multicolumn{4}{|l|}{ Accumbens } \\
\hline $\begin{array}{l}\text { Ipsilateral, lesion }(n=8) \\
\text { mean } \pm \text { SEM }\end{array}$ & $80.7 \pm 5.0$ & $1.7 \pm 0.3$ & $2.3 \pm 0.6$ \\
\hline $\begin{array}{l}\text { Contralateral, lesion }(n= \\
\text { 7) mean } \pm \text { SEM }\end{array}$ & $60.1 \pm 5.7$ & $2.4 \pm 0.9$ & $5.0 \pm 2.6$ \\
\hline \multicolumn{4}{|l|}{ Olfactory tubercle } \\
\hline Ipsilateral, mean \pm SEM & $46.8 \pm 4.1$ & $2.3 \pm 0.8$ & $4.5 \pm 1.4$ \\
\hline SEM & $46.7 \pm 5.3$ & $3.1 \pm 0.9$ & $6.6 \pm 2.3$ \\
\hline & $123.8 \pm 2.9$ & $0.4 \pm 0.1$ & 0.3 \\
\hline
\end{tabular}

water were available ad libitum. Each rat had a bipolar nichrome electrode (Plastic Products, MS-303-0.005 in.) implanted stereotaxically while under pentobarbital anesthesia. The electrode was aimed at the $A_{10}$ region of the ventral tegmentum, using the following coordinates: $2.8 \mathrm{~mm}$ anterior, $0.6 \mathrm{~mm}$ lateral, and $2.1 \mathrm{~mm}$ dorsal to stereotaxic 0 (Kopf). The stereotaxic incisor bar was set at $3.0 \mathrm{~mm}$ ventral to the interaural line.

Testing for self-stimulation was conducted in 6 Plexiglas boxes $(30 \times$ $30 \times 24 \mathrm{~cm})$ housed within sound-attenuating chambers. Depression of a lever $(4.5 \times 7.5 \mathrm{~cm})$ delivered AC sine wave current $(60 \mathrm{~Hz})$ of a fixed duration $(200 \mathrm{msec})$ through a flexible lead connected to the chronically implanted electrode assembly. The current intensity varied from 6 to $30 \mu \mathrm{A}$ and was set at a level that maintained consistent leverpressing during an initial $5 \mathrm{~d}$ scrcening pcriod. Only those animals displaying stable self-stimulation behavior were incorporated into the main experiment. Rate-intensity curves were determined over a 4 d period prior to unilateral 6-OHDA lesions. Current intensities were preset by a computer (Nova-3; Manx software) controlling the value of a set of resistors in a 6 -channel constant current stimulator. The current intensities were incremented in $2 \mu \mathrm{A}$ steps from an initial value of 6 $\mu \mathrm{A}$ to $30 \mu \mathrm{A}$. Ten free pulses of stimulation were delivered to each animal during the first minute of testing at a given current level. The number of bar presses was recorded for the subsequent 5 min period, after which the current value was set at the next level. Data collection was controlled by the computer and individual rate-intensity curves were plotted daily for each subject.

In preparation for the 6-OHDA lesions of the ascending DA pathways, either ipsilateral or contralateral to the stimulating clectrode, cach animal was anesthetized with halothane. Prior to surgery, the animals were given intraperitoneal injections of desipramine $\mathrm{HCl}(25 \mathrm{mg} / \mathrm{kg})$, a procedure that blocks damage to noradrenergic neurons by 6-OHDA (Roberts et al., 1975). The lesions were made by inserting a 34-gauge cannula into the ascending DA pathways at the level of the lateral hypothalamus. Lesion coordinates, with the incisor bar at $4.2 \mathrm{~mm}$ below the interaural line were $5.9 \mathrm{~mm}$ anterior, $2.1 \mathrm{~mm}$ lateral and dorsal to the interaural line. With the cannula in place, $4 \mu \mathrm{g}$ 6-OHDA hydrobromide (dosage expressed as the base and dissolved in $0.15 \mathrm{M} \mathrm{NaCl}$ containing $0.2 \mathrm{mg} /$ $\mathrm{ml}$ ascorbic acid) was injected in a volume of $2 \mu \mathrm{l}$ over a $10 \mathrm{~min}$ period.

Following a 10-14 d postoperative recovery period, each subject was tested again for self-stimulation, as measured by rate-intensity curves. Postlesion testing continued daily for 18 test sessions. Upon completion of behavioral testing, the animals were killed by cervical fracture. Their brains were rapidly removed, cut in the coronal plane at the level of the optic chiasm, mounted on a microtome, and frozen. Coronal sections (approximately $1 \mathrm{~mm}$ thick) were cut and from these the olfactory tubercle, nucleus accumbens, and striatum were dissected from both hemispheres. Biochemical analyses of DA and its metabolites, 3,4-dihydroxyphenylacetic acid (DOPAC) and homovanillic acid (HVA), were determined by high-pressure liquid chromatography (HPLC) with electrochemical detection according to Jakubovic et al. (1987). In bricf, the tissues were sonicated for $30 \mathrm{sec}$ in $0.2 \mathrm{M} \mathrm{HClO}_{4}$ containing $0.15 \%$ $\mathrm{Na}_{2} \mathrm{~S}_{2} \mathrm{O}_{5}$ and $0.5 \% \mathrm{Na}_{2}$ EDTA. Following centrifugation at $30,000 \times g$ for 15 min $\left(+4^{\circ} \mathrm{C}\right)$, the supernatant was removed and stored at $-80^{\circ} \mathrm{C}$. The HPLC system consisted of a Beckman model 100A pump, LC-4B amperometric controller with a glassy carbon electrode (BAS), a 3390A recorder integrator (Hewlett-Packard), and a $\mu$ Bondapak C18 column $(10 \mu \mathrm{m}, 150 \times 3.9 \mathrm{~mm}$; Waters). The detector potential was set at +0.75 $\mathrm{V}$ versus a $\mathrm{Ag}-\mathrm{AgCl}$ reference electrode, and the sensitivity was set at $10-20 \mathrm{nA} / \mathrm{V}$ full-scale. The mobile phase consisted of $0.1 \mathrm{M} \mathrm{KH}_{2} \mathrm{PO}_{4}$ buffer solution ( $\mathrm{pH} 3.6$ ) containing $0.2 \mathrm{~mm}$ sodium octanesulfonic acid, $0.15 \mathrm{mM} \mathrm{Na}_{2}$ EDTA, and $9.5 \%$ methanol. The concentrations of DA and its metabolites were determined by comparing the peak area of the sample with that of a known standard. The detection limit of the system was approximately $0.5 \mathrm{pmol} / 20 \mu \mathrm{l}$ of tissue sample.

The remaining midbrain tissue was saved for histological confirmation of the VTA electrode placement. This tissue was immersed in a $10 \%$ formalin solution for at least $48 \mathrm{hr}$, after which frozen sections ( 30 $\mu \mathrm{m}$ ) were taken in the coronal plane, mounted, and stained with cresyl violet prior to microscopic examination.

\section{Results and Discussion}

The unilateral 6-OHDA lesions produced extensive depletions of DA in the nucleus accumbens, olfactory tubercle, and striatum (Table 1). The greatest depletions were observed in the striatum, with DA concentrations in the lesioned hemisphere reduced to less than $1 \%$ of the values in the control hemisphere. DA levels in the nucleus accumbens and olfactory tubercle varied between 2 and $6 \%$ of control values. Of importance was the fact that the magnitude of depletion was similar whether the lesioned hemisphere was ipsilateral or contralateral to the stimulation electrode in the VTA.

The placements of the stimulation electrodes are shown in Figure 1, and the tip of each electrode was found to be in close proximity to the dopaminergic cell bodies that comprise the $A_{10}$ region of the VTA. The portion of mesencephalon containing the electrode was dissected incorrectly for one animal in the contralateral lesion group and the histological analysis could not be completed for this subject.

Figure 2 illustrates the effects of unilateral 6-OHDA lesions of mesotelencephalic DA pathways on the rate-intensity curves from VTA electrodes. In the ipsilateral lesion group, a 2-way repeated-measures ANOVA $(F(15,105)=5.99, p<0.01)$ of the bar-pressing data confirmed a significant interaction between current intensity and test day. Post hoc comparisons were conducted using Duncan's multiple-range test and these revealed that postoperative responding was significantly reduced at 14 $\mu \mathrm{A}$ and above $(p<0.05)$. In contrast, the identical analysis in the contralateral group indicated that there were no significant differences in the rate-intensity curves following 6-OHDA lesions in the contralateral hemisphere $(F(15,90)=0.82, p>$ $0.05)$.

On the basis of ICS rate-stimulus frequency functions, Miliaressis et al. (1986) have suggested that changes in the reinforcing efficacy of brain stimulation can be inferred from the shift in the number of rectangular pulses required at zero $\left(\Theta_{0}\right)$ and half-maximal $\left(\mathrm{M}_{50}\right)$ performance, with the latter measure providing the more accurate index of reward efficacy. Although a sine wave stimulation current was used in the present study and current intensity $(\mu \mathrm{A})$, rather than pulse frequency, was varied, analysis of the present data in terms of $M_{50}$ and $\Theta_{0}$ indicates that the ipsilateral lesions significantly reduced both 
A 2.18

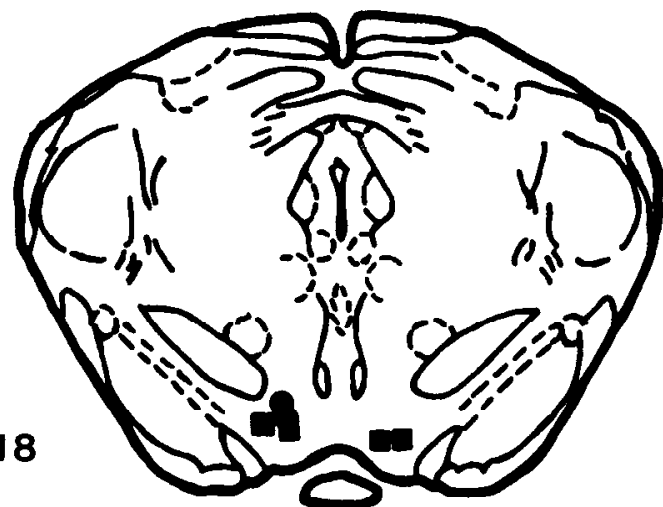

A 1.95

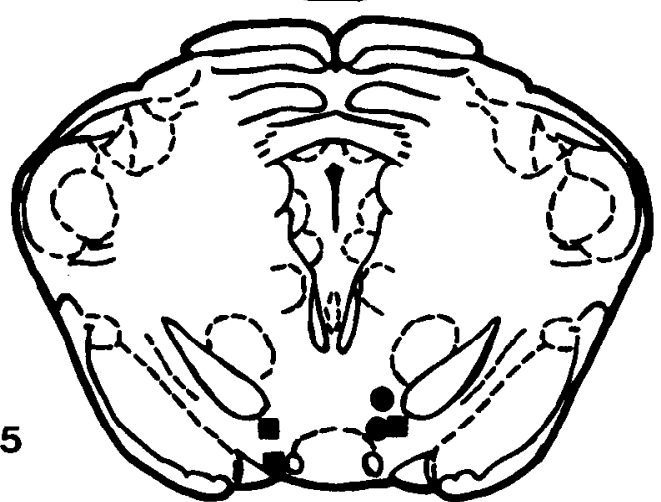

A 1.76

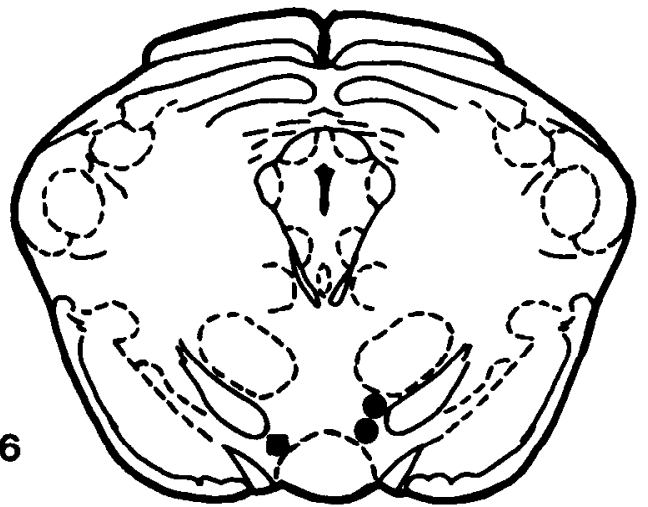

Figure 1. VTA electrode placements in ipsilateral and contralateral 6-OHDA lesion groups.

of these measures of reinforcement efficacy. Specifically, the current in microamperes required to maintain half-maximal rates of responding increased by between 43 (day 18 ) and $74 \%$ (day 3) after the ipsilateral lesions $(F(3,18)=4.59, p=0.015)$. In contrast, contralateral lesions did not affect the $\mathrm{M}_{50}$ values $(F(3,18)=0.49$, n.s. $)$ at any time after the lesions. Changes in current intensities required to maintain self-stimulation rates above a threshold of 100 bar presses $/ 5$ min (the definition of $\Theta_{0}$ chosen for the present study) were also compared following ipsilateral or contralateral lesions (Fig. 3). The prelesion values for the ipsilateral and contralateral groups were $\bar{X}=10.5 \pm 0.5$ $\mu \mathrm{A}$ and $\bar{X}=10.6 \pm 0.8 \mu \mathrm{A}$, respectively. A repeated-measures ANOVA on the threshold intensities of both groups revealed significant main effects for Group $(F(1,13)=5.23, p<0.05)$, Test $(F(3,30)=4.40, p<0.01)$, and a significant Group $\times$ Test interaction $(F(3,39)=3.59, p<0.05)$. Post hoc tests showed that the threshold intensities were significantly increased following the ipsilateral lesions and remained significantly higher

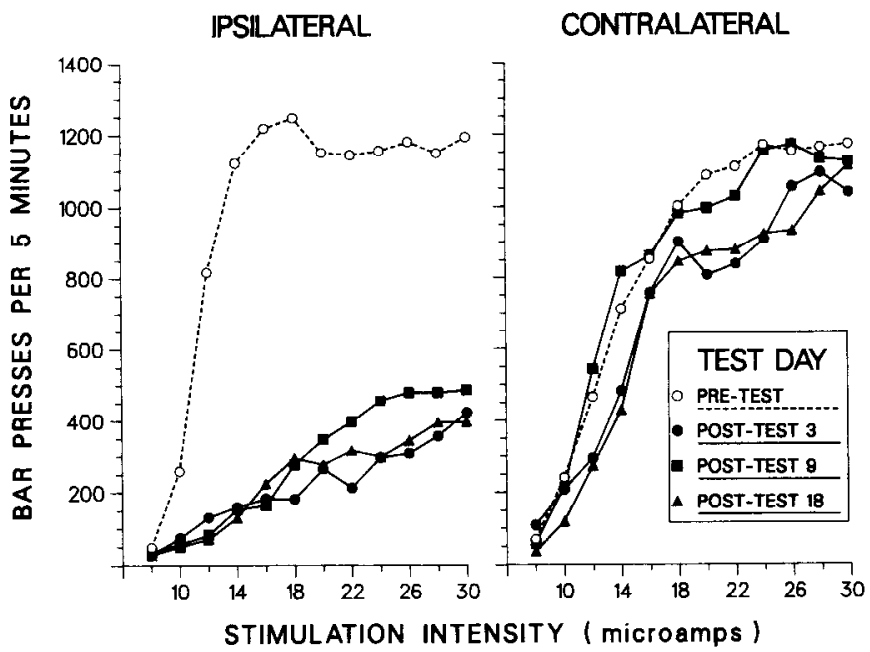

Figure 2. Effects of ipsilateral or contralateral 6-OHDA lesions of ascending mesotelencephalic DA projections on ICS rates obtained from the VTA at various stimulation intensities. ICS rate-current intensity functions were determined before and at various intervals after the 6-OHDA lesions. Data represent lever-pressing rates of 8 animals with ipsilateral lesions and 7 animals with contralateral lesions.

over the $18 \mathrm{~d}$ postlesion test period. No significant differences were observed between pre- and postlesion self-stimulation threshold intensities in the group receiving 6-OHDA lesions in the hemisphere contralateral to the VTA electrodes.

The present results show that extensive unilateral depletion of forebrain DA is accompanied by a significant disruption of self-stimulation in the ipsilateral VTA, as measured by changes in rate-intensity functions and by current intensities required to maintain threshold $\left(\Theta_{0}\right)$ and half-maximal $\left(\mathbf{M}_{50}\right)$ response rates. According to these measures, therefore, the ipsilateral lesions significantly decreased the reinforcing efficacy of the brain stimulation. In addition to this, however, it is noteworthy that the ipsilateral lesions resulted in highly significant decreases in the asymptotic rates of responding (Fig. 2). Several authors have

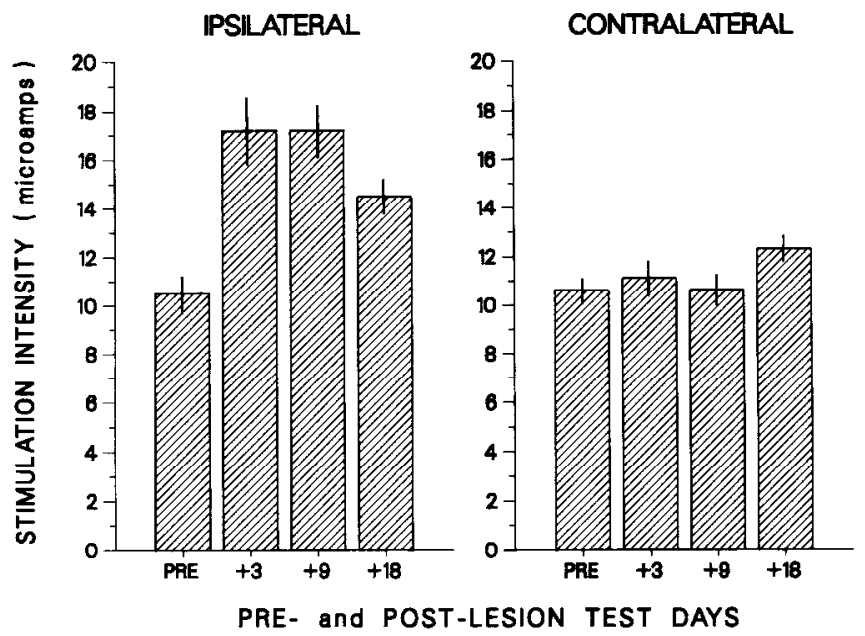

Figure 3. Effects of ipsilateral or contralateral 6-OHDA lesions of ascending mesotelencephalic DA projections on the current intensity required to maintain ICS rates above a threshold of 100 lever-presses for 5 min (see text for details). Data represent mean current intensities $(\mu \mathrm{A})$ of 8 animals with ipsilateral lesions and 7 animals with contralateral lesions. 
suggested that decreases in the asymptotic rate of responding indicate that the experimental manipulation in question has interfered with the performance capability of the animal (Edmonds and Gallistel, 1974; Miliaressis, 1986). According to this formulation, therefore, the ipsilateral lesions also resulted in motoric impairments that interfered with high rates of leverpressing. However, identical contralateral lesions failed to affect the ICS rate-current intensity function, including the asymptotic ratc of responding. If ipsilateral lesions produced motoric impairments, then similar deficits should also have been produced by the contralateral lesions. Because this was not observed, it is possible that changes in asymptotic rates of responding may not under all circumstances only reflect a change in the performance capacity of an animal. As damage to the contralateral mesotelencephalic DA systems failed to produce significant changes in ICS, the data support an important role for DA in mediating the rewarding properties of electrical stimulation of the VTA. As such, these data confirm earlier studies with electrodes in the vicinity of DA cell bodies in the VTA (Phillips and Fibiger, 1978) and with axons in the lateral hypothalamus (Koob et al., 1978).

Although the reduction in bar-pressing was substantial following ipsilateral 6-OHDA lesions, residual self-stimulation behavior was still obtained at the higher current intensities and this has important implications for understanding the neural substrates of reward in the VTA. There is evidence for separate dopaminergic (Phillips et al., 1982; Franklin and Vaccarino, 1983) and nondopaminergic (Clavier and Fibiger, 1977) reward pathways in this region of the brain, and activation of one or more of these nondopaminergic systems may have sustained some self-stimulation after the ipsilateral lesions. It is also possible that the few DA neurons that survived the 6-OHDA lesions may have been sufficient to maintain some degree of ICS.

\section{Experiment 2}

Several laboratories have shown that electrical stimulation of mesotelencephalic DA neurons increases DOPAC and HVA concentrations in the terminal regions of these neurons (Korf et al., 1976; Roth et al., 1976; McCown et al., 1983). However, these studies were conducted in anesthetized animals, and the relevance of the stimulation parameters to those used in ICS from the VTA is not clear. In the present experiment, it was reasoned that if DA neurons are activated by ICS electrodes aimed at the VTA, then the metabolites of DA should be increased in the terminal regions of these neurons.

\section{Materials and Methods}

Twenty-five hooded, Long-Evans male rats weighing $300-400 \mathrm{gm}$ werc implanted with electrodes aimed at the VTA. Stereotaxic coordinates with the incisor bar at $3.0 \mathrm{~mm}$ below the interaural line were $2.8 \mathrm{~mm}$ anterior, $0.6 \mathrm{~mm}$ lateral, and $2.1 \mathrm{~mm}$ dorsal to stereotaxic 0 . Two weeks after surgery, each subject was screened for self-stimulation. After 10 min of shaping, the rat was allowed to self-stimulate for $30 \mathrm{~min}$. The current intensity for each rat was selected to elicit the highest selfstimulation rate possible. This procedure was continued until each rat had at least $5 \mathrm{~d}$ of experience with self-stimulation. At this time rats were assigned to one of 3 groups: ICS $(n=9)$, Yoked Control $(n=4)$, and Unstimulated Control $(n=12)$.

Subjects in the ICS group had self-stimulation rates of 1785-3665 presses $/ 30 \mathrm{~min}$ at intensities ranging between 16 and $26 \mu \mathrm{A}$. The Yoked Control group had rates and intensities that matched those of 4 rats of the ICS group (950-3157 presses at 16-28 $\mu \mathrm{A}$ ). The Unstimulated Control group consisted of a "handled" subgroup $(n=5)$, and included 2 rats that showed little self-stimulation during screening, while 3 other animals had rates similar to those of the ICS group (2969-3621 presses at $20-26 \mu \mathrm{A})$. A second, "unhandled," subgroup $(n=7)$ included 5 rats that showed poor self-stimulation and 2 that were similar to those of the ICS group (2199-3230 presses at 20-24 $\mu \mathrm{A}$ ).

On the day after the last screening session, each rat in the ICS group was killed by cervical fracture immediately after a 30 min self-stimulation session. The brain was rapidly removed, placed on ice, dissected, and assayed by HPLC for DA, DOPAC, and HVA by the method of Jakubovic et al. (1987). Each rat in the Yoked Control group and its appropriate matched animal in the ICS group were placed in a separate ICS apparatus at the same time. No lever was available, and the brain stimulation received by each yoked animal was determined by the corresponding ICS rat. Thus, animals in the Yoked group did not determine the rate at which they received stimulation. Instead, they received brain stimulation at exactly the same rate as was generated by the corresponding member in the ICS group. Another difference between the ICS and Yoked group was that, on the test day, the current intensity used for each animal was the one that generated the highest ICS rate for that animal during the screening sessions. The largest difference in current intensity between a matched ICS-Yoked pair of animals was $4 \mu \mathrm{\Lambda}$. $\Lambda \mathrm{fter}$ 30 min of stimulation, rats in the Yoked Control group were killed and their brains processed as described above. On the test day, rats in the "handled" Unstimulated Control subgroup were placed in their usual ICS test box for 30 min with a stimulation lead connected, but no lever available and no stimulation delivered. They were then killed and processed as described above. Rats in the "unhandled" subgroup were taken from their home cages and killed without being placed in the ICS chamber.

\section{Results and Discussion}

The neurochemical data were analyzed by 2-way ANOVA with 1 between-group and 1 within-group factor, and by post hoc tests (Tukey's). The "handled" and "unhandled" subgroups of the Unstimulated Control group did not differ on any of the neurochemical measures, and therefore their data were combined. Compared to the Unstimulated Controls, electrical stimulation of the VTA did not affect the concentration of DA in any of the 3 terminal regions examined. Electrical stimulation of the VTA did, however, result in significant increases in DOPAC and HVA in a number of instances. For reasons of space, and because the ratios of HVA and/or DOPAC to DA have been suggested as being appropriate indices of DA utilization and turnover, the results are presented and discussed in terms of these ratios. It should be emphasized that because electrical stimulation did not affect DA concentrations, significant changes in these ratios were due primarily to changes in the concentrations of HVA and DOPAC.

For all 3 brain regions, the Group $\times$ Hemisphere interaction was significant for both DOPAC:DA and HVA:DA ratios $(p<$ 0.05 ). Subsequent pairwise comparisons indicated that compared to the Unstimulated Control group, ICS obtained from electrodes in the VTA produced significant increases in both DOPAC:DA and HVA:DA ratios in the ipsilateral striatum, nucleus accumbens, and olfactory tubercle (Figs. 4, 5). These increases ranged from a low of $25 \%$ in the DOPAC:DA ratio in the olfactory tubercle to a high of $64 \%$ in the HVA:DA ratio in the nucleus accumbens. Previous studies have reported significant increases in DA turnover in restricted regions of the forebrain, ipsilateral to ICS electrodes. Stimulation at sites in the VTA of the rat brain has been reported to be accompanied by an increased DOPAC:DA ratio, but only in the olfactory tubercle (Mitchell et al., 1982). Increased DA turnover in both the nucleus accumbens and rostral striatum of 2 inbred strains of mice has been observed after ICS at placements in the ventral region of the lateral hypothalamus (Garrigues and Cazala, 1983). McCown et al. (1986) have recently reported that ICS obtained from electrodes in the lateral hypothalamus does not increase 

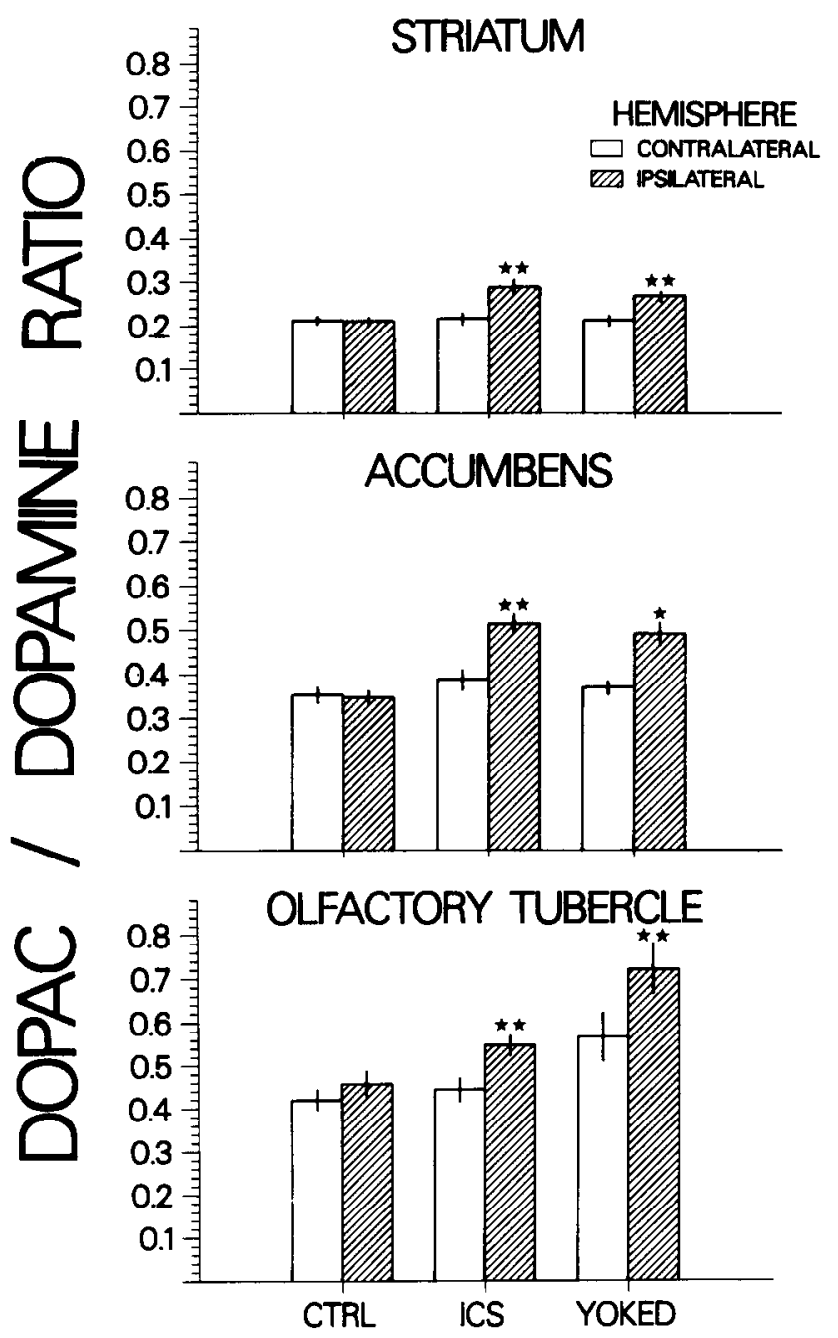

Figure 4. Effects of ICS obtained from electrodes in the VTA on the ratios of HVA to DA in the striatum, nucleus accumbens, and olfactory tubercle either ipsilateral or contralateral to the stimulating electrode. Control animals (CTRL; $n=12)$ were implanted with electrodes but did not receive brain stimulation on the day that they were killed. The ICS group $(n=9)$ was killed immediately after 30 min of self-stimulation. Animals in the Yoked Control group $(n=4)$ received brain stimulation at the same current intensity and rate as matched animals in the ICS group and were killed after $30 \mathrm{~min}$ of imposed brain stimulation. *Significantly different from contralateral side, $p<0.05$; ${ }^{* *} p$ $<0.01$.

DOPAC concentrations in the striatum, nucleus accumbens, or olfactory tubercles. However, these authors failed to note that their DOPAC:DA ratios were increased by $47 \%$ in the nucleus accumbens and by more than $100 \%$ in the olfactory tubercle of the self-stimulating animals. The ratio effect in the nucleus accumbens was confined to the side ipsilateral to the electrode, while in the olfactory tubercle it was bilateral. Inasmuch as ratio measures are more sensitive and appropriate indices of DA utilization than are metabolite concentrations, the data of McCown et al. (1986) are not incompatible with a role of DA neurons in ICS.

The increases in HVA:DA and DOPAC:DA ratios in the ICS group were confined to the side of the brain that was ipsilateral to the ICS electrode. Thus, in no case was there a significant difference between the ratios obtained on the side contralateral to the electrode in the ICS animals and the values obtained in
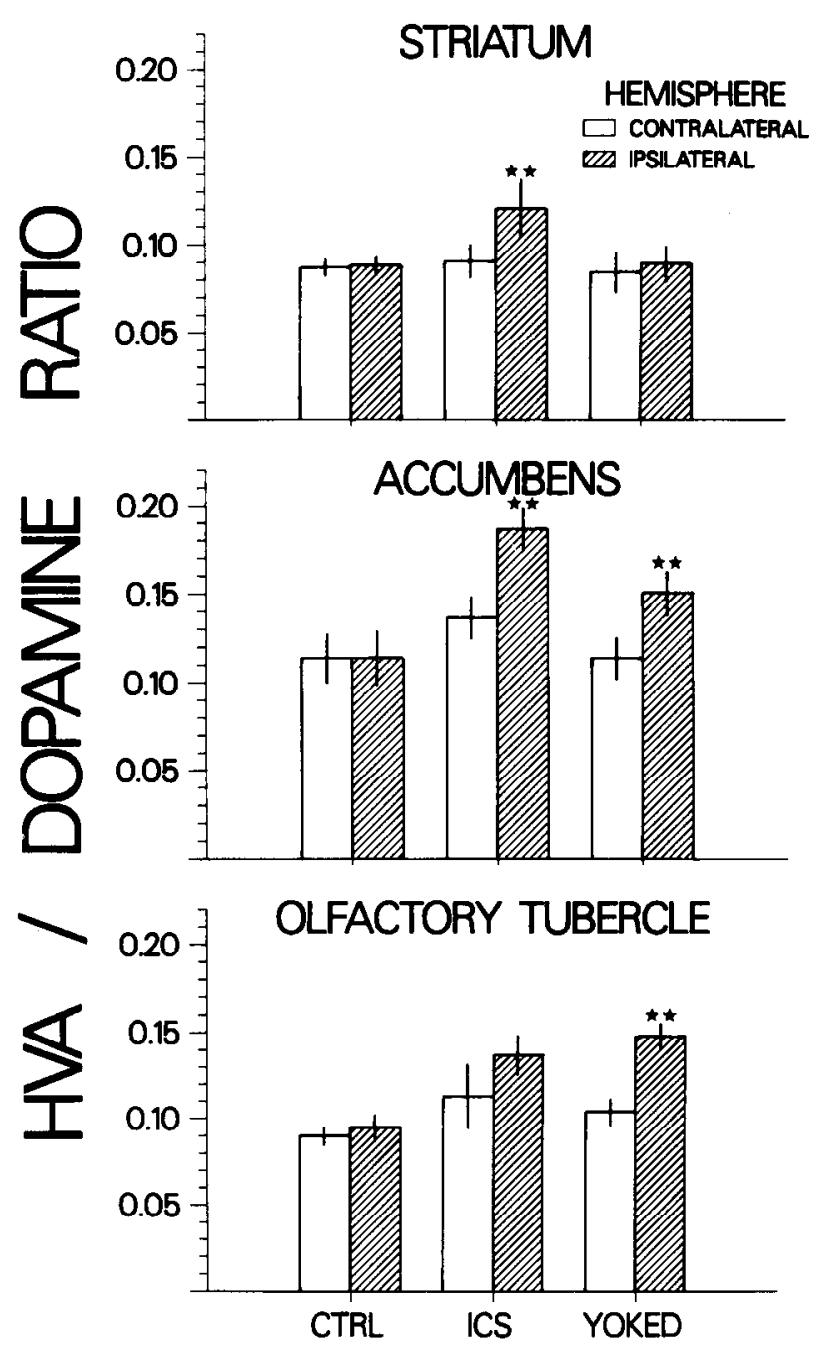

Figure 5. Effects of ICS obtained from electrodes in the VTA on the ratios of HVA to DA in the striatum, nucleus accumbens, and olfactory tubercle either ipsilateral or contralateral to the stimulating electrode. Control animals ( $C T R L ; n=12)$ were implanted with electrodes but did not receive brain stimulation on the day that they were killed. The ICS group $(n=9)$ were killed immediately after 30 min of self-stimulation. Animals in the Yoked Control group $(n=4)$ received brain stimulation at the same current intensity and rate as matched animals in the ICS group and were killed after $30 \mathrm{~min}$ of imposed brain stimulation. * Significantly different from contralateral side, $p<0.01$.

the Unstimulated Control group (Figs. 4, 5). This indicates that, to the extent that there was current spread from the ICS electrode, this was not sufficient to activate DA neurons in the contralateral VTA. More important, this finding indicates that the increases in DA metabolism observed in the ipsilateral forebrain regions of the ICS group were a consequence of electrical stimulation of the DA neurons, rather than a reflection of the high level of operant behavior exhibited by these animals. Given the nature of the operant task, motor-related changes in DA metabolism should have been evident in both sides of the brain.

The data obtained from the Yoked Control group further indicate that the neurochemical changes observed in the ICS group were not a result of the operant behavior displayed by these animals. The yoked animals received brain stimulation at identical rates and similar currents as did the ICS animals. They did not, however, lever-press for this stimulation. Despite this, electrical stimulation of the VTA resulted in neurochemical 
changes in the Yoked group that were very similar to those obtained in the ICS animals. Specifically, in contrast to unstimulated controls, in all but one instance, stimulation of the VTA in the yoked animals resulted in significant increases in both HVA:DA and DOPAC:DA ratios in the ipsilateral striatum, nucleus accumbens, and olfactory tubercles (Figs. 4, 5). The one negative finding was that yoked stimulation did not increase the HVA:DA ratio in the ipsilateral striatum despite increasing the DOPAC:DA ratio in this structure by a significant $26 \%$. The factors responsible for this negative result are not known.

It is of interest that yoked stimulation resulted in neurochemical effects that were very similar to those observed after ICS, because previous research has demonstrated that yoked stimulation can be aversive (Steiner et al., 1969). Also, using 2-deoxy$\mathrm{D}-{ }^{14} \mathrm{C}$-glucose autoradiography, Porrino et al. (1984) have shown that ICS and yoked stimulation can produce different patterns of metabolic activation in the forebrain. It remains to be determined whether the stimulation received by the yoked animals in the present study was reinforcing or aversive, and such studies are planned.

\section{Experiment 3}

The previous experiment demonstrated that ICS obtained from electrodes in the VTA results in ipsilateral increases in DA utilization in the striatum, nucleus accumbens, and olfactory tubercle. The fact that DA metabolism did not change in the contralateral hemisphere, as well as the data obtained in the Yoked Control animals, suggests that these effects were not due to the high rates of operant behavior displayed by the ICS animals. The present experiment had 2 purposes. The first was to evaluate further the possibility that high rates of operant behavior may accelerate DA metabolism in DA-rich forebrain regions, that is, to provide further control data pertinent to the performance issues discussed in Experiment 2. The second goal was to determine whether a reinforcer other than brain stimulation of the VTA would increase DA metabolism in these forebrain structures. To this end, the effects of high rates of leverpressing for food on DA metabolism were investigated.

\section{Materials and Methods}

Thirty-six male hooded rats (450-500 gm) of the Long-Evans strain were food-deprived to $85-90 \%$ of their body weights. Twelve rats were shaped to lever-press for food pellets ( $45 \mathrm{mg}$; Noyes) on a continuous reinforcement schedule (CRF) for $30 \mathrm{~min} / \mathrm{d}$. After $5 \mathrm{~d}$ on the CRF schedule, the density of reinforcement was gradually decreased until the animals worked on a schedule in which every eighth lever-press was reinforced (FR8). Lever-pressing was maintained on this schedule for 10-12 d. At the same time that the above animals were tested each day, animals from a second group $(n=12)$ were placed in adjoining chambers in which the levers had been removed. An animal in this group (Yoked Controls) automatically received a food pellet each time that its leverpressing counterpart was rewarded with food. Animals in the third group (Unfed Controls; $n=12$ ) were placed in an adjacent chamber without levers simultaneous with the Lever-Pressing group. However, animals in this group never received food pellets in the operant chamber. Thus, there were 12 squads, consisting of 3 animals each: a lever-presser, a yoked control, and an unfed control. The members of each squad remained constant throughout the experiment. One hour after each daily session, each rat received an amount of laboratory chow in its home cage sufficient to maintain its weight at the appropriate level.

On the test day, animals from each squad were killed by cervical fracture immediately after the $30 \mathrm{~min}$ behavioral session. The brains were rapidly removed, dissected, and analyzed for DA and its metabolites by HPLC, as described in Experiment 1.

\section{Results and Discussion}

Animals in the Lever-Pressing group responded for food at a mean rate of $1682 \pm 145$ lever-presses/30 min on the test day. This is substantially less than the mean rate of $2883 \pm 109$ lever-presses/ 30 min emitted by the ICS group on the test day in Experiment 2, but nevertheless represents a high level of operant behavior.

There were no significant differences in the concentrations of DA, DOPAC, and HVA in the striatum and nucleus accumbens between the Lever-Pressing, Yoked, and Unfed Control groups. Neither did the DOPAC:DA ratios in the nucleus accumbens and striatum differ between the 3 groups (Fig. 5). However, in the nucleus accumbens the HVA:DA ratios differed significantly between the 3 groups $(F(2,33)=3.92, p<0.05)$. Post hoc comparisons indicated that the HVA:DA ratio was higher in the Yoked group than in either the Lever-Pressing or the Unfed Control group (Fig. 6). The failure to find increased DOPAC: DA or HVA:DA ratios in the lever-pressing animals suggests that, compared to the unfed, food-deprived rats, those leverpressing for food do not have an increase in DA metabolism in the nucleus accumbens or striatum. It is recognized, however, that this conclusion assumes that the efflux rates of DOPAC and HVA from these structures did not differ between the 3 groups. Heffiner et al. (1984) have recently reported that the transport of DOPAC and HVA from the brain is enhanced during the performance of operant behavior. Furthermore, these authors have observed increased metabolism of DA in the striatum, but not in the nucleus accumbens, following lever-pressing for water reinforcement (Seiden and Heffner, 1984). This being the case, the present negative findings in the nucleus accumbens and striatum of the Lever-Pressing group must be interpreted with caution. The failure to observe effects on DA metabolism in the food-reinforced animals may have also been related to their lower rates of responding compared to the ICS subjects in Experiment 2. However, this can be discounted, as DA metabolism was not significantly affected in the contralateral regions of the ICS animals (Figs. 4, 5). Nevertheless, it remains possible that differences in the density of reinforcement between the 2 situations may have contributed to the failure to observe effects in the food-rcinforced group.

The neurochemical results were somewhat different in the olfactory tubercle. Specifically, the concentration of HVA differed significantly between the 3 groups $(F(2,33)=5.75, p<$ 0.01 ). Post hoc comparisons revealed that this was due to the HVA values being significantly higher in the Lever-Pressing and Yoked groups than in the Unfed Control group $(p<0.05$ in both instances). Group differences in the HVA:DA ratios were not statistically significant $(F(2,33)=2.62, p=0.09)$. Figure 7 shows that the HVA:DA ratios tended to be higher in the LeverPressing and the Yoked Control groups. Thus, in comparison to unfed controls, the HVA:DA ratios were increased by $15 \%$ in the Lever-Pressing group and by $28 \%$ in the Yoked Control group. These data indicate that, as compared to unfed, fooddeprived controls, there was a trend towards enhanced DA metabolism in both the Lever-Pressing and the Yoked Control groups. From this it may be concluded that food consumption, rather than lever-pressing, was responsible for the increased concentration of HVA in the olfactory tubercles and for the trend towards enhanced DA turnover in this structure. The extent to which these findings are associated with the reinforcing properties of food, as opposed to the sensory and motor func- 
Figure 6. Effects of lever-pressing for food on an FR8 schedule of reinforcement on the ratio of DOPAC to DA in the striatum, nucleus accumbens, and olfactory tubercle. Data from the left and right brain region from each animal were pooled. Control animals (CTRL; $n=12$ ) were not trained to lever-press for food and never received food pellets in the operant chamber, but were otherwise treated identically to the other groups. Lever-pressing animals ( $L E$ $V E R ; n=12$ ) were killed immediately after $30 \mathrm{~min}$ of lever-pressing for food. Animals in the Yoked group $(n=12)$ did not lever-press but automatically received food each time that their counterpart in the Lever group was rewarded with food. See text for details.

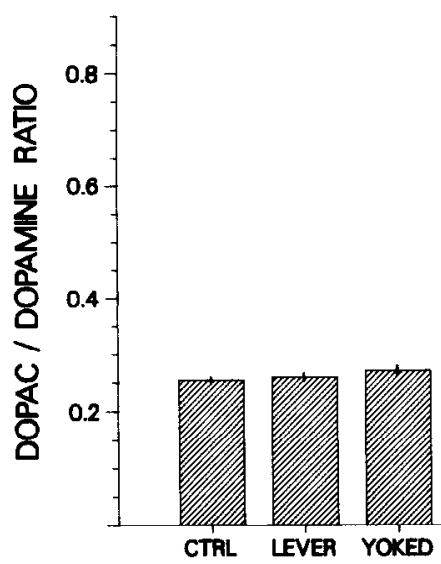

ACCUMBENS OLFACTORY TUBERCLE

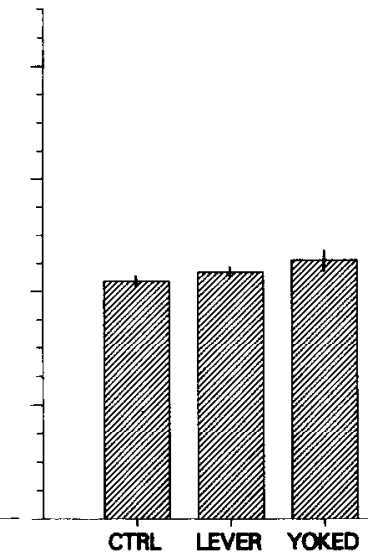

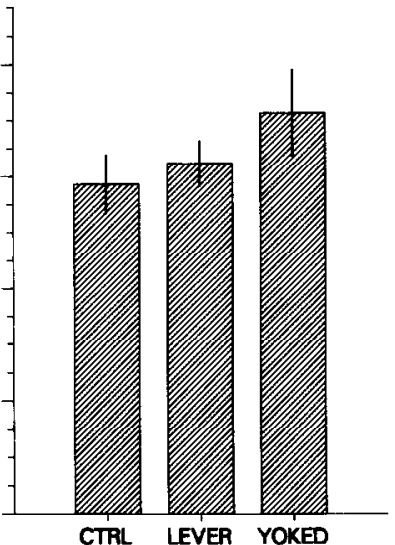

tions associated with food consumption, remains to be determined.

\section{General Discussion}

The major findings of this study provide strong support for a role for DA neurons in ICS obtained from the VTA. In the first experiment, selective lesions of the ascending mesotelencephalic DA projections produced marked decreases in the reinforcing properties of brain stimulation when the lesion was ipsilateral to the stimulating electrode. Lesions of the contralateral DA projections were without significant effect. The second experiment demonstrated that ICS obtained from electrodes in the VTA resulted in highly significant increases in DOPAC:DA and HVA:DA ratios in the striatum, nucleus accumbens, and olfactory tubercle. These effects were confined to the side ipsilateral to the stimulating electrode, indicating that they were due to the electrical stimulation, rather than being associated with the high motor output that was displayed by these animals. The third experiment confirmed that high rates of operant behavior maintained by a different reinforcer (food) were not associated with significant changes in DA metabolism, as estimated by DOPAC:DA and HVA:DA ratios.

The finding that 6-OHDA lesions of the ipsilateral mesotelencephalic projection at the level of the lateral hypothalamus decreased ICS obtained from the VTA extends our previous work, in which only one stimulation current was used (Phillips and Fibiger, 1978). This observation confirms that there is an important role for ascending DA projections in ICS obtained from electrodes in the VTA. However, despite the extensive depletions of forebrain DA, some ICS was still demonstrated by these animals (Fig. 2). This suggests that there are non-DA systems in the vicinity of the VTA that are capable of supporting moderate levels of ICS, among which is perhaps the descending myelinated system that has been studied by Gallistel et al. (1981) and Gratton and Wise (1985). Alternatively, the few ascending DA axons that survived the 6-OHDA lesion may have been sufficient to maintain postlesion ICS. Previously, we have shown that 6-OHDA lesions similar to those used here produced only transient effects on ICS obtained from the substantia nigra (Clavier and Fibiger, 1977). Also, ICS obtained from the prefrontal cortex and nucleus accumbens is little affected by extensive lesions of the mesolimbic DA system (Phillips and Fibiger, 1978; Simon et al., 1979). These and the present observations indicate that while components of the mesotelencephalic DA system are important for ICS obtained from the VTA, this is not the case for other regions of the brain from which ICS can be obtained. As such, these observations fail to support the hypothesis that one of the ascending DA projection systems is a final common path for rewarding effects of all kinds (Wise, 1982). Instead, it appears that a DA system, most probably the mesolimbic pro-
Figure 7. Effects of lever-pressing for food on an FR8 schedule of reinforcement on the ratio of HVA to DA in the striatum, nucleus accumbens, and olfactory tubercle. Data from the left and right brain region from each animal were pooled. Control animals (CTRL; $n=$ 12) were not trained to lever-press for food and never received food pellets in the operant chamber but were otherwise treated identically to the other groups. Lever-pressing animals ( $L E$ $V E R ; n=12$ ) were killed immediately after $30 \mathrm{~min}$ of lever-pressing for food. Animals in the Yoked group $(n=12)$ did not lever-press but automatically received food each time that their counterpart in the Lever group was rewarded with food. See text for details. * Significantly different from control group, $p<$ 0.05 .

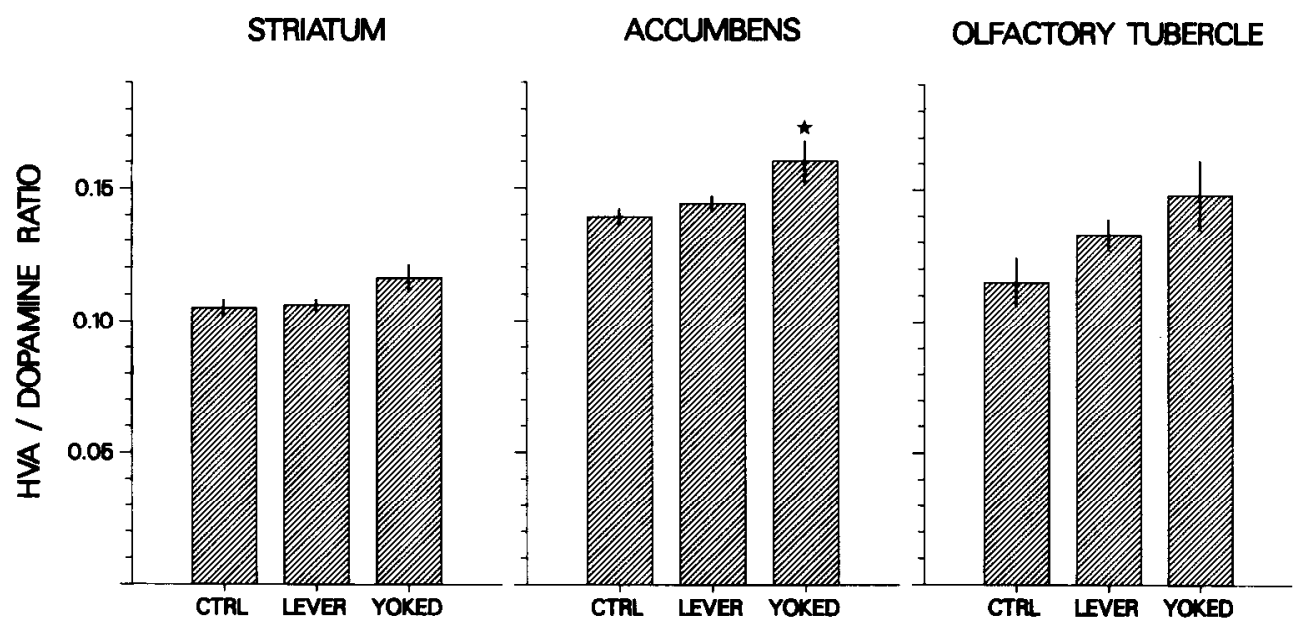


jection (see below), is but one of a number of reward-related systems in the brain. Electrophysiological data are consistent with the conclusion that there are multiple reward-related fibers capable of supporting ICS (Schenk and Shizgal, 1982). Also, the observation that ICS electrodes in the VTA, but not in the posterior hypothalamus, increase DA metabolism in the olfactory tubercle is compatible with a nonessential role for mesolimbic DA systems in brain-stimulation reward (Mitchell et al., 1982).

Many pharmacological studies of brain-stimulation reward have employed the curve-shift paradigm to dissociate impaired performance from drug modulation of a reward mechanism (e.g., Miliaressis et al., 1986). According to this perspective, the downward shift in the rate-intensity curves observed in the present study after ipsilateral lesions (Fig. 2) could be interpreted as a performance deficit. However, it should be emphasized that the curve-shift paradigm is not ideally suited to situations in which there is a near-total removal of a neuroanatomical substrate of reward, as is the case with 6-OHDA lesions of the mesotelencephalic DA pathways. Logically, a marked reduction in the level of brain-stimulation reward should be accompanied by a significant attenuation of lever-pressing rates, as was observed here. Miliaressis et al. (1986) have argued that the most sensitive index of change in the magnitude of brain-stimulation reward is the threshold, or $\Theta$, value, and this measure was increased significantly following ipsilateral, but not contralateral, 6-OHDA lesions. The fact that contralateral lesions failed to affect $\Theta$ values, $\mathbf{M}_{50}$, or asymptotic rates of lever-pressing provides strong evidence against performance effects following the unilateral 6-OHDA lesions employed in the present study. Nor can it be argued that ipsilateral lesions cause selective deficits in performance that are specific to stimulation on one side of the brain. Elsewhere we have reported that similar 6-OHDA lesions selectively attenuate ICS at sites in the VTA, while failing to have lasting effects on ICS obtained from the nucleus accumbens in the same hemisphere (Phillips and Fibiger, 1978).

Brain-stimulation reward obtained from the VTA increased DOPAC:DA and HVA:DA ratios in the striatum, nucleus accumbens, and olfactory tubercle. Recently, increased in vivo tyrosine hydroxylase activity has been observed in these same regions after ICS from electrodes in the VTA (Phillips et al., 1987). Three previous investigations have failed to observe significant effects of electrical stimulation in the region of the VTA on DA metabolism or release in terminal regions of the mesotelencephalic DA system (Miliaressis et al., 1975; Saint-Laurent et al., 1975; Myers and Mora, 1977). However, Simon et al. (1979) have provided cogent criticisms of these studies, which need not be reiterated here. In contrast, the present results are fully consistent with findings of Simon et al. (1979), who reported that ICS from the VTA increased DOPAC:DA ratios in the nucleus accumbens and prefrontal cortex. It appcars, therefore, that ICS obtained from electrodes in the VTA increases DA utilization in all of the major terminal regions of the mesotelencephalic DA systems.

Data such as these raise the question of which of these terminal regions are most important for mediating the rewarding properties of the brain stimulation. In what is perhaps the definitive pharmacological study on this point, Mogenson et al. (1979) found that unilateral injections of the DA receptor antagonist spiroperidol into the nucleus accumbens significantly reduced ICS of the ipsilateral VTA, but did not affect the rate of ICS obtained from the contralateral VTA. The contrasting effects of the ipsilateral and contralateral nucleus accumbens injections on VTA ICS indicates that spiroperidol-induced motor deficits cannot account for these observations. The specificity of the effect of the ipsilateral nucleus accumbens injections was also demonstrated by the finding that injections of spiroperidol into either the ipsilateral or contralateral prefrontal cortex did not affect VTA ICS. Therefore, in contrast to those in the nucleus accumbens, DA receptors in the prefrontal cortex do not appear to be important for mediating the rewarding effects of VTA ICS. Data obtained with the intravenous self-administration paradigm also indicate that DA terminals in the nucleus accumbens serve important reward-related functions. When these terminals are lesioned with 6-OHDA, there is a marked decrease in intravenous self-administration of indirect DA agonists, such as cocaine and $d$-amphetamine (Lyness et al., 1979; Roberts et al., 1980; Pettit et al., 1984). In contrast, 6-OHDA lesions of the medial prefrontal cortex do not affect intravenous self-administration of cocaine (Martin-Iverson et al., 1986).

Gallistel et al. (1985) have shown that it is possible to obtain relatively high rates of ICS from electrodes in the posterior hypothalamus and anterior VTA using stimulation parameters that do not result in metabolic activation of the striatum, nucleus accumbens, and olfactory tubercles, as measured by 2-deoxy$\mathrm{D}-{ }^{14} \mathrm{C}$-glucose autoradiography. This is, perhaps, not surprising, since Millar et al. (1985) have shown that the stimulation parameters used by Gallistel et al. (1985) (i.e., squarewave pulses of $0.1 \mathrm{msec}$ duration) do not depolarize DA axons, as measured by DA release in the striatum. When stimulation parameters appropriate for stimulation-induced release of DA were employed (i.e., $>0.5 \mathrm{msec}$ duration), Gallistel et al. (1985) were able to observe metabolic activation in the nucleus accumbens and olfactory tubercle ipsilateral to the stimulating electrode. It is possible, therefore, that differences between the stimulation parameters used in the present study and that of Gallistel et al. (1985) may have contributed to the different conclusions. Nevertheless, the data offered by Gallistel et al. (1985) indicate that it is possible to obtain robust ICS without activation of the mesotelencephalic DA systems. This raises the question of whether the enhanced DA turnover produced by VTA ICS in the present experiments was causally related to the rewarding properties of the brain stimulation or was an epiphenomenon incidental to the brain-stimulation reward.

Several considerations indicate that the ascending DA projections of the VTA have an important role in mediating the rewarding effects of ICS obtained from this site. First, ipsilateral lesions of the ascending DA projections resulted in marked decreases in VTA ICS that could not be attributed to lesion-induced motor deficits. Second, as noted above, there is strong evidence that the rewarding properties of VTA ICS and of intravenously self-administered stimulants such as $d$-amphetamine and cocaine are mediated by the mesolimbic DA projection (Lyness et al., 1979; Mogenson et al., 1979; Roberts et al., 1980; Pettit et al., 1984). These data indicate that enhanced DA release from terminals of the mesolimbic DA system, be it produced by electrical stimulation or by drugs, is sufficient to produce reward. However, the data of Gallistel et al. (1985) indicate that stimulation of other systems can also be rewarding and that these systems can function independently of the mesolimbic DA system. Thus, the DA system studied here and the non-DA system(s) identified by Gallistel et al. (1985) appear to function in parallel rather than being connected in series. Our results do not support the conclusion of Gallistel et al. (1985), 
that "ascending catecholaminergic pathways are not the directly stimulated component of the reward pathway" (p. 1257). Rather, the evidence reviewed above indicates that there is not a single reward pathway, that the mesolimbic DA projection is but one link in a reward-related system, and that there exist other reward circuits that do not appear to contain this DA link.

\section{References}

Bielajew, C., and P. Shizgal (1982) Behaviorally derived measures of conduction velocity in the substrate for rewarding medial forebrain bundlc stimulation. Brain Res. 237: 107-119.

Clavier, R. M., and H. C. Fibiger (1977) On the role of ascending catecholaminergic projections in self-stimulation of the substantia nigra. Brain Res. 131: 271-286.

Edmonds, D. E., and C. R. Gallistel (1974) Parametric analysis of self-stimulation reward in the rat: III. Effect of performance variables on the reward summation functions. J. Comp. Physiol. Psychol. 87: 876-883.

Fibiger, H. C., and A. G. Phillips (1986) Reward, motivation and cognition: Psychobiology of mesotelencephalic dopamine systems. In Handbook of Physiology: The Nervous System IV, F. E. Bloom and S. R. Geiger, eds., pp. 647-675, American Physiological Society, Bethesda, MD.

Franklin, K. B. J., and F. J. Vaccarino (1983) Differential effects of amphetamine isomers on SN self-stimulation: Evidencc for DA ncuron subtypes. Pharmacol. Biochem. Behav. 18: 747-751.

Gallistel, C. R., P. Shizgal, and J. S. Yoemans (1981) A portrait of the substrate for self-stimulation. Psychol. Rev. 88: 228-273.

Gallistel, C. R., Y. Gomita, E. Yadin, and K. A. Campbell (1985) Forebrain origins and terminations of the medial forebrain bundle metabolically activated by rewarding stimulation or by reward blocking doses of pimozide. J. Neurosci. 5: 1246-1261.

Carrigues, A.-M., and P. Cazala (1983) Central catecholamine metabolism and hypothalamic self-stimulation behaviors in two inbred strains of mice. Brain Res. 265: 265-271.

Gratton, A., and R. A. Wise (1985) Hypothalamic reward mechanism: Two first-stage fiber populations with a cholinergic component. Science 227: 545-548.

Heffner, T. G., M. Vosmer, and L. S. Seiden (1984) Increased transport of 3,4-dihydroxyphenylacetic acid from brain during performance of operant behavior in the rat. Brain Res. 293: 85-91.

Jakubovic, A., D. Fu, and H. C. Fibiger (1987) Factors affecting the stability and separation of biogenic amines and their metabolites: Simultaneous measurement by HPLC with electrochemical detection. J. Pharmacol. Methods 17: 1-14.

Koob, G. F., P. J. Fray, and S. D. Iversen (1973) Self-stimulation at the lateral hypothalamus and locus coeruleus after specific unilateral lesions of the dopamine system. Brain Res. 146: 123-140.

Korf, J., L. Grasdijk, and B. H. C. Westerink (1976) Effects of electrical stimulation of the nigrostriatal pathway of the rat on dopamine metabolism. J. Neurochem. 26: 579-584.

Lyness, W. H., N. M. Friedle, and K. E. Moore (1979) Destruction of dopaminergic nerve terminals in the nucleus accumbens: Effect on $d$-amphetamine self-administration. Pharmacol. Biochem. Behav. 11: 663-666.

Martin-Iverson, M. T., C. Szostak, and H. C. Fibiger (1986) 6-Hydroxydopamine lesions of the medial prefrontal cortex fail to influence intravenous self-administration of cocaine. Psychopharmacology 88 : 310-314

McCown, T. J., R. B. Mueller, and G. R. Breese (1983) The effects of anesthetics and electrical stimulation on nigro-striatal dopaminergic neurons. J. Pharmacol. Exp. Ther. 224: 489-493.

McCown, T. J., T. C. Napier, and G. R. Breese (1986) Effects of chronic electrode implantation in dopaminergic ncurons in vivo. Pharmacol. Biochem. Behav. 25: 63-69.

Miliaressis, E., N. B. Thoa, Y. Tizabi, and D. M. Jacobowitz (1975) Catecholamine concentration of discrete brain areas following selfstimulation of the ventro-medial tegmentum of the rat. Brain Res. 100: 192-197.

Miliaressis, E., P.-P. Rompre, P. Laviolette, L. Philippe, and D. Cou- lombe (1986) The curve-shift paradigm in self-stimulation. Physiol. Behav. 37: 85-91.

Millar, J., J. A. Stamford, Z. L. Kruk, and R. M. Wightman (1985) Electrochemical, pharmacological and electrophysiological evidence of rapid dopamine release and removal in the rat caudate nucleus following electrical stimulation of the median forebrain bundle. Eur. J. Pharmacol. 109: 341-348.

Mitchell, M. J., N. M. Nicolaou, G. W. Arbuthnott, and C. M. Yates (1982) Increases in dopamine metabolism are not a general feature of intracranial self-stimulation. Life Sci. 30: 1081-1085.

Mogenson, G. J., M. Takigawa, A. Robertson, and M. Wu (1979) Selfstimulation of the nucleus accumbens and ventral tegmental area of Tsai attenuated by microinjections of spiroperidol into the nucleus accumbens. Brain Res. 171: 247-259.

Myers, R. D., and F. Mora (1977) In vivo neurochemical analysis, by push-pull perfusion of the mesocortical dopaminergic system of the rat during self-stimulation. Brain Res. Bull. 2: 105-112.

Pettit, H. D., A. Ettenberg, F. E. Bloom, and G. F. Koob (1984) Destruction of dopamine in the nucleus accumbens selectively attenuates cocaine but not heroin self-administration in rats. Psychopharmacology 84: 167-173.

Phillips, A. G. (1984) Brain reward circuitry: A case for separate systems. Brain Res. Bull. 12: 195-201.

Phillips, A. G., and H. C. Fibiger (1978) The role of dopamine in maintaining intracranial self-stimulation in the ventral tegmentum, nucleus accumbens and medial prefrontal cortex. Can. J. Psychol. 32: 58-66.

Phillips, A. G., F. G. LePiane, and H. C. Fibiger (1982) Effects of kainic acid and lesions of the striatum on self-stimulation in the substantia nigra and ventral tegmental area. Behav. Brain Res. 5: 297-310.

Phillips, A. G., A. Jakubovic, and H. C. Fibiger (1987) Increased in vivo tyrosine hydroxylase activity in rat telencephalon produced by self-stimulation of the ventral tegmental area. Brain Res. 402: 109116.

Porrino, L. J., R. V. Esposito, T. F. Seeger, A. M. Crane, A. Pert, and L. Sokoloff (1984) Metabolic mapping of the brain during rewarding self-stimulation. Science 224: 306-309.

Roberts, D. C. S., A. P. Zis, and H. C. Fibiger (1975) Ascending catecholamine pathways and amphetamine-induced locomotor activity: Importance of dopamine and apparent non-involvement of norepinephrine. Brain Res. 93: 441-454.

Roberts, D. C. S., G. F. Koob, P. Klonoff, and H. C. Fibiger (1980) Extinction and recovery of cocaine self-administration following 6hydroxydopamine lesions of the nucleus accumbens. Pharmacol. Biochem. Behav. 12: 781-787

Roth, R. H., L. C. Murrin, and J. R. Walters (1976) Central dopaminergic neurons: Effects of alterations in impulse flow on the accumulation of dihydroxyphenylacetic acid. Eur. J. Pharmacol. 36: 163-171.

Saint-Laurent, J., M. F. Roizen, E. Miliaressis, and D. M. Jacobowitz (1975) The effects of self-stimulation on the catecholamine concentration of discrete areas of the rat brain. Brain Res. 99: 194-200.

Schenk, S., and P. Shizgal (1982) The substrates for lateral hypothalamic and medial prefrontal cortex self-stimulation have different refractory periods and show poor spatial summation. Physiol. Behav. 28: 133-138.

Seiden, L. S., and T. G. Heffner (1984) Alteration of brain catecholamine metabolism by environmental and behavioral events: An explanation of drug-behavior interactions. In Catecholamines: Neuropharmacology and Central Nervous System-Therapeutic Aspects, E. Usdin, ed., pp. 275-284, Liss, New York.

Simon, H., L. Stinus, J. P. Tassin, S. Lavielle, G. Blanc, A.-M. Thierry, J. Glowinski, and M. Le Moal (1979) Is the dopaminergic mesocorticolimbic system necessary for intracranial self-stimulation? Biochemical and behavioral studies from A10 cell bodies and terminals. Behav. Neural Biol. 27: 125-145.

Steiner, S. S., B. Beer, and M. M. Shaffer (1969) Escape from selfproduced rates of brain stimulation. Science 163: 90-91.

Wise, R. A. (1982) Neuroleptics and operant behavior: The anhedonia hypothesis. Behav. Brain Sci. 5: 39-87. 